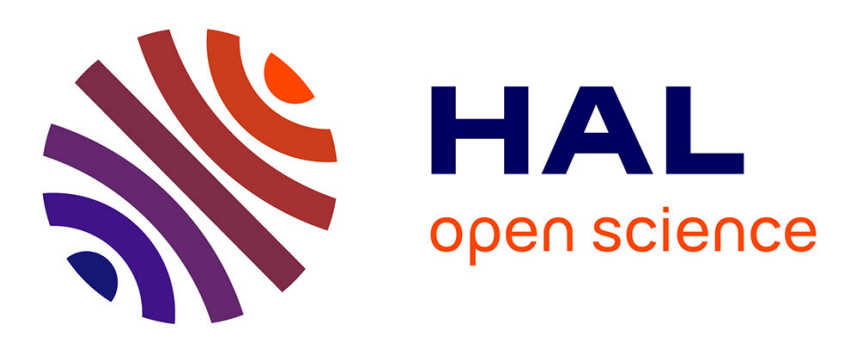

\title{
Probing Time-Dependent Molecular Dipoles on the Attosecond Time Scale
}

\author{
C. Neidel, J. Klei, C.-H. Yang, A. Rouzee, M. J. J. Vrakking, K. Klunder, M. \\ Miranda, C.L. Arnold, T. Fordell, A. L'Huillier, et al.
}

\section{- To cite this version:}

C. Neidel, J. Klei, C.-H. Yang, A. Rouzee, M. J. J. Vrakking, et al.. Probing Time-Dependent Molecular Dipoles on the Attosecond Time Scale. Physical Review Letters, 2013, 111, pp.033001. 10.1103/PhysRevLett.111.033001 . hal-00990233

\section{HAL Id: hal-00990233 https://hal.science/hal-00990233}

Submitted on 11 Feb 2021

HAL is a multi-disciplinary open access archive for the deposit and dissemination of scientific research documents, whether they are published or not. The documents may come from teaching and research institutions in France or abroad, or from public or private research centers.
L'archive ouverte pluridisciplinaire HAL, est destinée au dépôt et à la diffusion de documents scientifiques de niveau recherche, publiés ou non, émanant des établissements d'enseignement et de recherche français ou étrangers, des laboratoires publics ou privés. 


\title{
Probing Time-Dependent Molecular Dipoles on the Attosecond Time Scale
}

\author{
Ch. Neidel, J. Klei, C.-H. Yang, A. Rouzée, and M. J. J. Vrakking* \\ Max-Born Institut, Max-Born Strasse 2A, 12489 Berlin, Germany \\ K. Klünder, M. Miranda, C. L. Arnold, T. Fordell, A. L’Huillier, M. Gisselbrecht, and P. Johnsson \\ Department of Physics, Lund University, P.O. Box 118, SE-221 00 Lund, Sweden \\ M.P. Dinh and E. Suraud \\ Laboratoire de Physique Théorique-IRSAMC, University Paul Sabatier Toulouse 3, \\ 118 Route de Narbonne, 31062 Toulouse Cedex, France \\ P.-G. Reinhard \\ Institut für Theoretische Physik, Universität Erlangen, Staudtstrasse 7, D-91058 Erlangen, Germany \\ V. Despré, M. A. L. Marques, and F. Lépine ${ }^{\dagger}$ \\ Institut Lumière Matière, Université Lyon 1, CNRS, UMR 5306, 10 Rue Ada Byron, 69622 Villeurbanne Cedex, France
}

(Received 21 March 2013; published 18 July 2013)

\begin{abstract}
Photoinduced molecular processes start with the interaction of the instantaneous electric field of the incident light with the electronic degrees of freedom. This early attosecond electronic motion impacts the fate of the photoinduced reactions. We report the first observation of attosecond time scale electron dynamics in a series of small- and medium-sized neutral molecules $\left(\mathrm{N}_{2}, \mathrm{CO}_{2}\right.$, and $\left.\mathrm{C}_{2} \mathrm{H}_{4}\right)$, monitoring time-dependent variations of the parent molecular ion yield in the ionization by an attosecond pulse, and thereby probing the time-dependent dipole induced by a moderately strong near-infrared laser field. This approach can be generalized to other molecular species and may be regarded as a first example of molecular attosecond Stark spectroscopy.
\end{abstract}

DOI: 10.1103/PhysRevLett.111.033001

PACS numbers: 33.80.Eh, 42.65.Ky

One of the most fascinating prospects in the emerging attosecond science field concerns real time observation and control of the electronic properties of matter. In recent years, attosecond laser pulses, synthesized by high harmonic generation [1] were used to unravel a wide range of phenomena in atoms [2-6], in small molecules [7,8], and on surfaces [9]. Because of the low available extreme ultraviolet (XUV) photon flux, experiments with an isolated attosecond pulse or an attosecond pulse train (APT) are generally performed in combination with a phase-locked, near-infrared (NIR), femtosecond (fs) pulse. Photoelectron spectroscopy [streaking [10,11] and RABITT (reconstruction of attosecond beating by interference of two- photon transitions) [12] measurements], ion momentum imaging [7,8], and transient absorption [5] have already been applied in experiments where the optical cycle of the NIR femtosecond pulse is used as a clock, thus allowing us to monitor the time evolution of processes, initiated or probed by the attosecond XUV pulse.

In molecules, all observed attosecond time scale effects that were measured so far were attributed to dynamics accompanying or following ionization $[8,11,13]$. However, several theoretical works have already described scenarios for inducing attosecond electron dynamics in neutral molecules [14,15]. For example, Burnus et al. [16] have shown how attosecond transient electron dynamics lead to electronic excitation. An approach that would allow the experimental observation of such dynamics remains to be developed.

Classically, the initial step of laser excitation corresponds to the acceleration of an electron by the light electric field, which creates a time-dependent dipole. This time-dependent dipole may both be created during the interaction with the light field, when the electron density responds to the electric force exerted by the light field, or after the interaction with the light field, if this interaction has led to the formation of a coherent superposition of electronic states. In the present case, we will consider the dipole created during the interaction of a molecule with a light field.

In this Letter, we report a two-color femtosecond NIR pump-attosecond XUV probe experiment in a series of small- to medium-sized neutral molecules $\left(\mathrm{N}_{2}, \mathrm{CO}_{2}\right.$, and $\mathrm{C}_{2} \mathrm{H}_{4}$ ), where the time-dependent dipole induced by the moderately strong, NIR laser field is monitored with attosecond time resolution. Pronounced oscillations are observed in the parent molecular ion yield as a function of the pump-probe delay. These oscillations are interpreted in terms of a time-dependent screening induced by the polarization of the molecule, which alters the photoionization yield of the neutral molecule. Our findings are supported by time-dependent density functional theory 
(a)

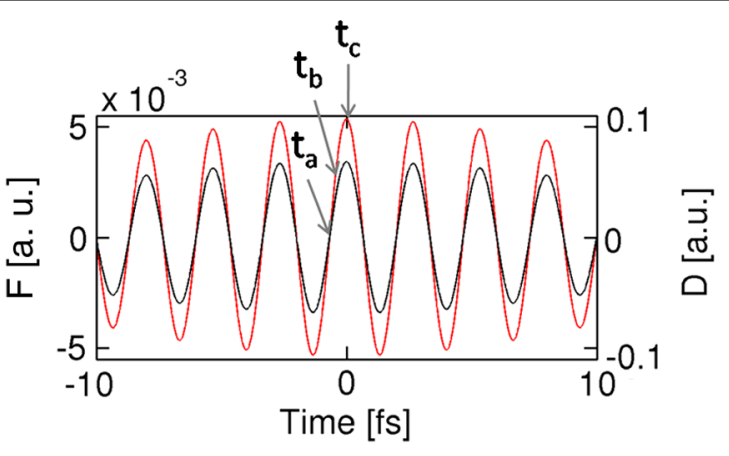

(b)

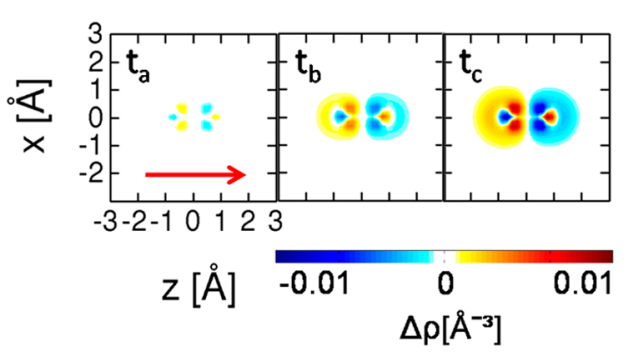

(c)
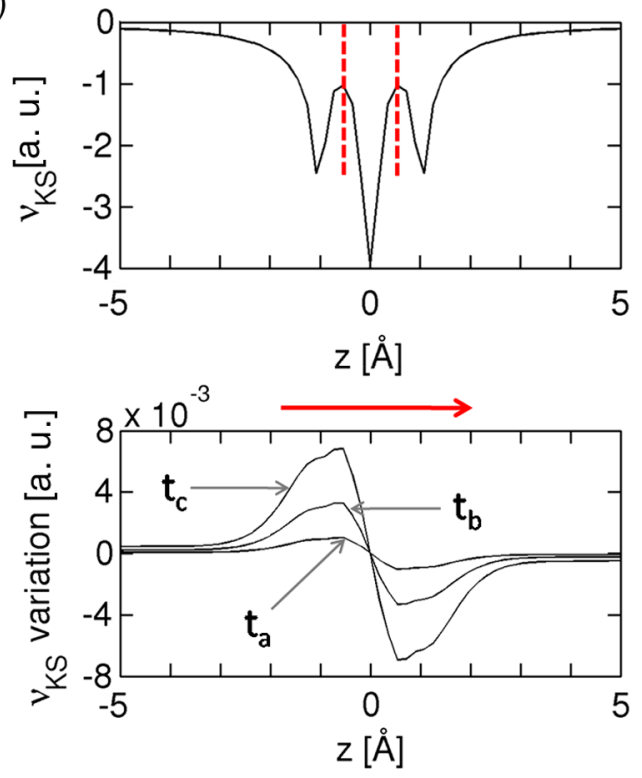

FIG. 1 (color online). (a) Calculated molecular dipole $D$ (black curve) induced by a NIR femtosecond laser field $F$ (thin red curve), for the case of an aligned $\mathrm{N}_{2}$ molecule interacting with a $30 \mathrm{fs}, 800 \mathrm{~nm}, 10^{12} \mathrm{~W} / \mathrm{cm}^{2}$ laser pulse. The NIR field induces an adiabatic time-dependent dipole that corresponds to a displacement of the charge density distribution around the fixed nuclei. (b) Field-induced electronic density changes at three different time delays and, hence, field strengths [see (a)]. Times chosen are $t_{a}=t_{0}+75$ as, $t_{b}=t_{0}+334$ as, $t_{c}=t_{0}+$ $667 \mathrm{as}$ ), where $t_{0}$ corresponds to the time of a zero-crossing of the laser electric field. The color scale indicates an increase (light red) or decrease (dark blue) of the electron density around the nuclei. The red arrow indicates the direction of the light electric field. (c) Top: Kohn-Sham potential $V_{\mathrm{KS}}$ for an aligned $\mathrm{N}_{2}$ molecule, where the position of the nuclei are shown with red dashed lines; (down) variation of the Kohn-Sham potential when an electric field is present. The Kohn-Sham potential acts as a screening field when the polarized molecule interacts with an ionizing XUV laser pulse (see Fig. 2).
(TDDFT) calculations using both the OCTOPUS [17] and the TELEMAN [18] packages. The calculation is achieved on a discretized grid. Core electrons are considered as frozen and are described, in addition with nuclei, by a Troullier Martins norm conserving pseudopotential. We have used the LDA-Sic (local density approximation with selfinteraction correction) functional. The details of the calculations will be presented elsewhere (see the Supplemental Material [19] and Ref. [20]). To illustrate how the electronic density in a neutral molecule is driven by an incident NIR laser electric field, calculations were performed for an $\mathrm{N}_{2}$ molecule interacting with a linearly polarized $800 \mathrm{~nm}$ laser field. The laser intensity was set to $10^{12} \mathrm{~W} / \mathrm{cm}^{2}$ to avoid noticeable ionization. In the chosen example an $\mathrm{N}_{2}$ molecule was considered with the molecular axis aligned along the light electric field. As shown in Fig. 1(a), the computed time-dependent dipole of the molecule (dark line) adiabatically follows the time-dependent light electric field (thin red curve). At the chosen wavelength and laser intensity, the electrons are simply driven by the instantaneous light electric field without significant population transfer to electronically excited states. Classically, the time-dependent variation of the dipole reflects an oscillatory motion of the electrons along the molecular axis. This is illustrated in Fig. 1(b), which shows the variation of the electron density (i.e., the difference between the electronic density with and without the laser field), at three different times [indicated in the upper curve in Fig. 1(a)], corresponding to three different laser field strengths. This leads to an asymmetric charge distribution, which generates the molecular dipole plotted in Fig. 1(a).

The time-dependent change of the electron localization around the nuclei induces changes in the interaction between the molecule and an attosecond XUV pulse ionizing the molecule. In the ionization process, the transition of one electron into the ionization continuum is determined by the XUV field and by the average potential that describes the interaction of the electron with all electrons and ions within the molecule. In terms of mean field theory, this additional potential corresponds to the Kohn-Sham potential [21]. The Kohn-Sham potential calculated for $\mathrm{N}_{2}$ along the molecular axis is plotted in Fig. 1(c), together with its variation under the influence of the NIR electric field. When the XUV light interacts with the polarized molecule, its effect on the molecule is screened by the Kohn-Sham potential, leading to either an increase or decrease of the ionization efficiency.

In Fig. 2, we consider the case where a, fixed-in-space, $\mathrm{N}_{2}$ molecule that is polarized by a femtosecond NIR pump pulse ( $800 \mathrm{~nm}, 30 \mathrm{fs}$ pulse duration, $10^{12} \mathrm{~W} / \mathrm{cm}^{2}$ ) interacts with an XUV APT (300 as individual pulse duration, $10^{9} \mathrm{~W} / \mathrm{cm}^{2}$, one pulse per NIR half-cycle, photon energy centered at $35 \mathrm{eV}$ ). For a given NIR-XUV delay, the ionization yield is calculated as the total electronic charge that is transferred into the continuum after $120 \mathrm{fs}$ of time propagation. The calculation is repeated for different 
(a)
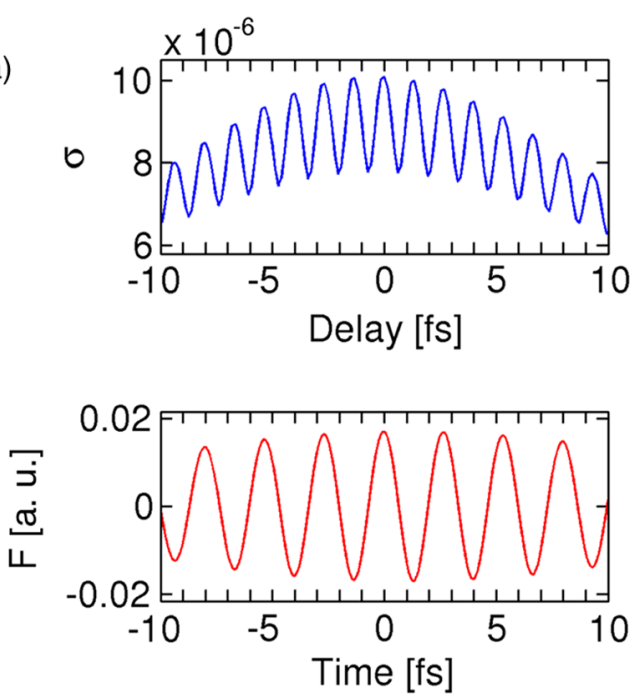

(b)
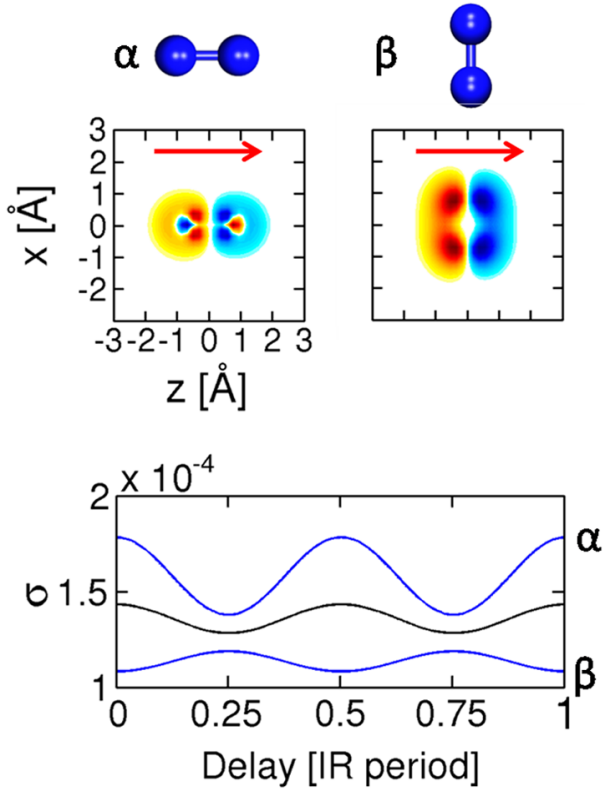

FIG. 2 (color online). (a) Ionization probability $\sigma$ plotted as a function of the NIR-XUV pump-probe delay (upper curve), along with the variation of the NIR electric field $F$ (lower curve). The latter time axis is plotted in such a way that the time axis corresponds to the instant where the APT is synchronized. The maximum ionization is observed when the APT is synchronized with the extrema of the NIR field. In this case, the calculation was performed in $2 \mathrm{D}$ in order to decrease the computational time. (b) Variation of the ionization probability $\sigma$, for two different cases of the molecular alignment (when the molecules are aligned along $(\alpha)$ or perpendicular $(\beta)$ to the laser polarization axis). Importantly, although showing a pronounced alignment dependence, the field-induced oscillation persists in the case of alignment-averaged calculations (middle, black curve).

NIR-XUV pump-probe delays leading to the upper curve presented in Fig. 2(a) [for comparison, the NIR electric field is shown in the lower half of Fig. 2(a)]. When the APT is synchronized to the extrema of the NIR electric field, the ionization yield is a maximal. Conversely, the ionization yield has a minimum when the APT is synchronized with the zero crossings of the laser electric field. Consequently, the total ionization yield oscillates as a function of the NIR-XUV delay with a period that is half the period of the NIR electric field. As discussed above, this oscillation is due to the influence of screening of the XUV light electric field by the NIR-induced polarization of the molecule, and as such is a manifestation of the time-dependent localization of the electrons within the molecule.

We note that variations of the ionization yield were previously observed in attosecond pump-probe experiments on helium atoms [22]. In these experiments, the bandwidth of the XUV light (with a $10 \mathrm{eV}$ wide spectrum centered around $25 \mathrm{eV}$ ) was partially below the field-free ionization threshold of helium $(24.58 \mathrm{eV})$, allowing the efficient population of excited states below the ionization threshold, which could subsequently be ionized by the NIR field. Here, we consider situations where the XUV photon energy is tuned well above the ionization threshold of the molecules (which is typically below $16 \mathrm{eV}$ ).

In gas phase experiments, laser pulses usually interact with randomly aligned molecules. The effect of molecular alignment is considered in Fig. 2(b). The amplitude of the NIR-XUV delay-dependent oscillations is noticeably decreased when the NIR and XUV pulses are polarized perpendicularly to the molecular axis (blue curve). Moreover, in this case, the yield oscillations are out of phase with the oscillations that occur for molecules aligned along the laser polarization, so that the maximum of ionization occurs at the zero of the NIR electric field. Importantly, the yield oscillations remain observable in alignment-averaged calculations [see middle black curve in Fig. 2(b)], albeit with a reduced amplitude.

To experimentally verify the possibility to observe the effects predicted above, an experimental arrangement containing a two-color NIR + XUV Mach-Zehnder-type interferometer and a mass spectrometric detection system was used (see Fig. 2 in the Supplemental Material [19]). A NIR laser beam $(800 \mathrm{~nm}, 30 \mathrm{fs}, 1 \mathrm{kHz})$ was split into two beams. One arm contained the NIR pump beam $(1 \mathrm{~mJ})$, whose intensity was chosen to avoid ionization of the target molecules (estimated to be roughly $10^{13} \mathrm{~W} / \mathrm{cm}^{2}$ ). An XUV APT was generated in the other arm, by focusing up to $2 \mathrm{~mJ}$ laser pulses into a gas cell containing argon. The cutoff of the XUV spectrum was around harmonic 31 $(48 \mathrm{eV})$. An aluminum thin film (300 $\mathrm{nm}$ thickness) was used to spectrally filter the NIR fundamental beam as well as harmonics with orders below 11 from the XUV APT (see Fig. 3 in the Supplemental Material [19]). The XUV probe beam was combined with the NIR pump beam using a mirror with a center hole [23]. Both the XUV probe and NIR pump beam were collinearly focused onto the molecular target by a toroidal mirror. The two-color experiment was carried out in the active region of a velocity map 
(a) Parent ion yield

(b) FT power spectrum

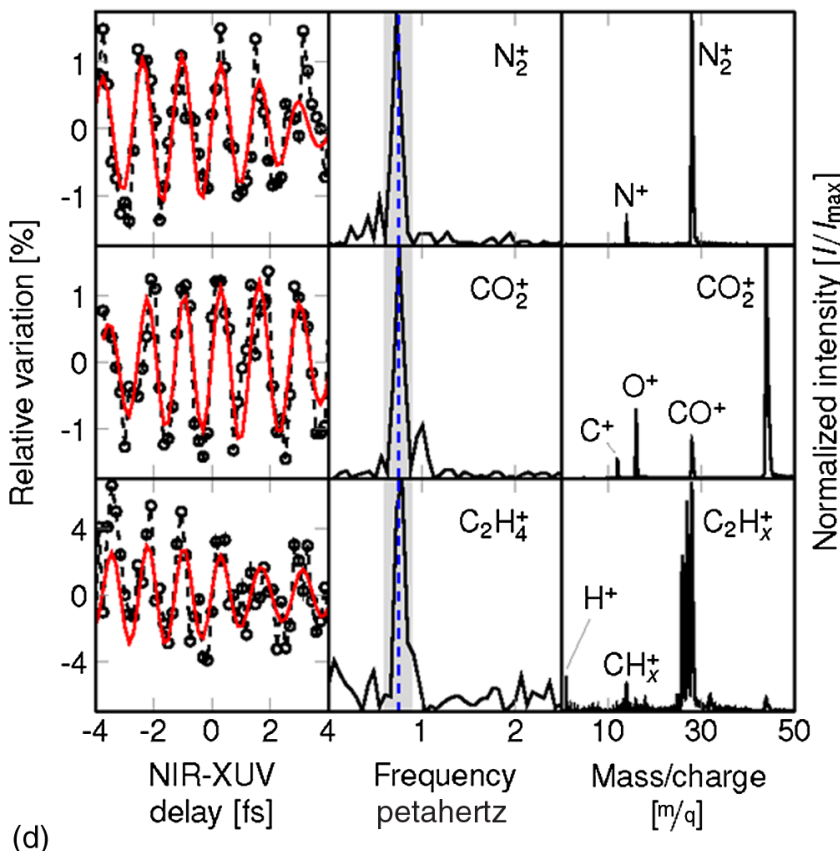

(d)

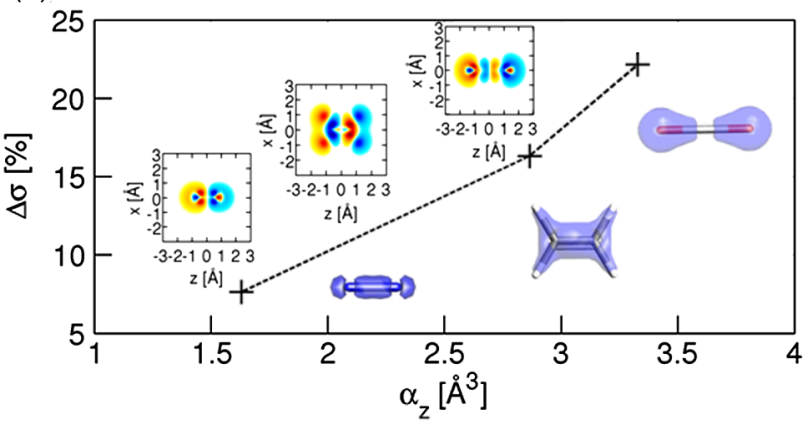

FIG. 3 (color online). (a) Experimentally measured $\mathrm{N}_{2}^{+}, \mathrm{CO}_{2}^{+}$, and $\mathrm{C}_{2} \mathrm{H}_{4}^{+}$yields (black dashed curves) as a function of the delay between the NIR pump and the XUVAPT probe generated in argon. These oscillations trace the IR induced time-dependent molecular dipole and therefore the attosecond electronic motion in the neutral molecule prior to ionization. The relative placement of the timeaxis of the three measurements is undefined, and has been chosen in a manner where for all three molecules a zero crossing in the yield occurs at $T=0$. (b) Normalized Fourier transform (FT) power spectra of the experimental results. The shaded gray area was used in an inverse-FT, leading to the red solid curves in the left panel. The parent ionization yields oscillate with a period corresponding to half the period of the NIR field (dashed blue line). (c) Time-of-flight spectra obtained for ionization of $\mathrm{N}_{2}, \mathrm{CO}_{2}$, and $\mathrm{C}_{2} \mathrm{H}_{4}$ molecules by an APT generated by high harmonic generation in argon. In each molecule the abundance of the parent ion dominates over that of the ionic fragments. (d) TDDFT calculations of the amplitude of the oscillation of the XUV-induced ionization yield $\Delta \sigma$, of aligned molecules, as a function of the polarizability tensor component along the molecular axis $\alpha_{z}$. The NIR-induced charge displacement increases with the polarizability of the molecule, enhancing the screening effect that leads to the ionization yield variations. The induced change in the electron density at the maximum of the NIR electric field is shown above the data points, while the ground state chemical bonding structure is shown to the right. imaging spectrometer [24]. The molecular target was introduced by means of a capillary [25] in the repeller electrode. Ions formed were accelerated toward a set of microchannel plates followed by a phosphor screen and a CCD camera. Illustrating the generality of the observations, ion yield measurements were made for $\mathrm{N}_{2}, \mathrm{CO}_{2}$, and $\mathrm{C}_{2} \mathrm{H}_{4}$.

The measurements consisted of recording the cationic species produced by XUV-induced ionization as a function of the delay between the XUVAPT and the NIR pulse which was phase locked to the XUV APT. The right column of Fig. 3 shows time-of-flight spectra recorded with the velocity map imaging spectrometer for the three molecular species $\left(\mathrm{N}_{2}, \mathrm{CO}_{2}\right.$, and $\left.\mathrm{C}_{2} \mathrm{H}_{4}\right)$ after interaction with the APT only. In $\mathrm{N}_{2}$, the ionization leads mainly to the formation of an intense parent ion peak where the molecular ion is formed in one of its three first ionic states, namely, the $X^{2} \Sigma_{g}^{+}$, $A^{2} \Pi_{u}$, and $B^{2} \Sigma_{u}^{+}$states. The XUV photon energies used in this experiment also enables access to the predissociative $C^{2} \Sigma_{u}^{+}$state and to the dissociative $F^{2} \Sigma_{g}^{+}$state, which leads to the observed $\mathrm{N}^{+}$ peak in the mass spectra. In $\mathrm{CO}_{2}$, three charged fragments are observed, as well as a main contribution from the $\mathrm{CO}_{2}^{+}$parent ion. More extensive fragmentation is observed in the case of the $\mathrm{C}_{2} \mathrm{H}_{4}$. For these molecules many states can be involved in the ionization or dissociation process. In the left column of Fig. 3, the variation of the singly charged parent molecular ions yield versus pump-probe delay is shown. In the middle column of Fig. 3, Fourier transform power spectra of these signals are shown as well, indicating the dominant frequency component. In the case of $\mathrm{N}_{2}^{+}$, an oscillation with a maximum modulation depth of about $1 \%-2 \%$ and a period equal to half the NIR laser period is observed. A similar in-phase oscillation was observed for $\mathrm{N}^{+}$, indicating that the total ionization yield oscillates as a function of the time delay between the APT and the NIR field. This is a strong indication that the NIR field polarizes the neutral molecule and that the transition induced by the APT is sensitive to the redistribution of the electronic density, as observed in our TDDFT calculation (see Fig. 2). We note that the amplitude of the experimental oscillation is smaller than the one calculated with TDDFT, which is likely due to the fact that the experiment involves averaging over the laser focal volume and the molecular alignment, and is furthermore influenced by nuclear degrees of freedom which were not included in the TDDFT calculation.

Similar experiments were performed for $\mathrm{CO}_{2}$ and $\mathrm{C}_{2} \mathrm{H}_{4}$, where oscillations with an average amplitude of $2.5 \%$ (for $\mathrm{CO}_{2}^{+}$) and about $6 \%\left(\mathrm{C}_{2} \mathrm{H}_{4}^{+}\right)$were observed, indicating the influence of the molecular polarizability tensor on the observed oscillations. In the case of randomly oriented molecules, we just consider the average scalar polarizability, and observe that for the chosen molecules the amplitude of the oscillations increases with their total polarizability: $\mathrm{N}_{2}\left(1.71 \AA^{3}\right), \mathrm{CO}_{2}\left(2.507 \AA^{3}\right), \mathrm{C}_{2} \mathrm{H}_{4}\left(4.18 \AA^{3}\right)$.

TDDFT calculations were performed on $\mathrm{CO}_{2}$ and $\mathrm{C}_{2} \mathrm{H}_{4}$ following the same protocol as previously described in the case of $\mathrm{N}_{2}$. In Fig. 3(d), the amplitude of the timedependent variation of the ionization yield resulting from two-color NIR + XUV excitation is plotted for $\mathrm{N}_{2}, \mathrm{CO}_{2}$, 
and $\mathrm{C}_{2} \mathrm{H}_{4}$ as a function of their calculated polarizability tensor component along the molecular axis. As in Fig. 2(a), the case of molecules aligned along the laser field axis is considered. The quasilinear dependence observed illustrates the fact that a higher polarizability leads to a higher variation of the ionization yield because the electrons are more efficiently displaced from their equilibrium position, thereby creating a larger screening potential. Please note that the polarizability along the molecular axis is higher for $\mathrm{CO}_{2}$ than for $\mathrm{C}_{2} \mathrm{H}_{4}$ (the order is reversed for the average polarizability). Interestingly, attosecond probing of NIRinduced molecular electron dynamics may be regarded as an ultrafast variant of molecular Stark spectroscopy, which is a well-established tool for extracting information on structure and chemical bonds in molecules. Molecular Stark spectroscopy provides direct information on the polarizability tensors of the molecular sample. We note that the possible advantage of the use of an intense laser field in Stark spectroscopy (enabling extensive control over the field strength, oscillation period, etc.) was already pointed out in Ref. [26]. Given that the observed NIR electric field-induced changes in the XUV ionization yield depend on detailed properties of the orbitals involved in the ionization (symmetry, ionization cross section, and polarizability), experiments such as the ones discussed in this Letter could be used to completely characterize the polarizability tensors of the states involved, especially when they are carried out with aligned molecules. This goal, as well as the application of the method to more complex systems will be pursued in the near future.

In conclusion, we have demonstrated that NIR-driven time-dependent dipoles in neutral molecules can be observed by means of variations of the ionization efficiency by an APT. The effect is understood in terms of a screening of the XUV field by the NIR-induced polarization. The amplitude of the attosecond oscillations is determined by the polarizability tensor of the electronic states involved in the absorption of the XUV light and therefore represents a first implementation of molecular Stark spectroscopy on the attosecond time scale. In the future, our approach could not only be used to measure timedependent molecular polarizability tensors in complex molecules but might also allow monitoring attosecond electronic motion during chemical reactions.

This research was supported by the Marie Curie program ITN ATTOFEL (FP7-PEOPLE-ITN-2008, Grant No. 238362), the European Research Council (ALMA), the Access program and Joint Research Activity ALADIN of Laserlab-Europe II, the Swedish Foundation for Strategic Research, the Swedish Research Council, the Knut and Alice Wallenberg Foundation, the European Initiative of Infrastructures, and the Sträng Foundation, ANR-10BLAN-0428-01, ANR-08-CEXC8-008-01, Institut Universitaire de France. F. L. would like to thank Laserlab
Europe for financial support and the Lund Laser Center where we carried out the initial experiment demonstrating the attosecond variation of the molecular ionization yield.

*Marc.Vrakking@mbi-berlin.de

${ }^{\dagger}$ franck.lepine@univ-lyon1.fr

[1] F. Krausz and M. Ivanov, Rev. Mod. Phys. 81, 163 (2009).

[2] P. Agostini and L. F. DiMauro, Rep. Prog. Phys. 67, 813 (2004).

[3] M. Drescher, M. Hentschel, R. Kienberger, M. Uiberacker, V. Yakovlev, A. Scrinzi, Th. Westerwalbesloh, U. Kleineberg, U. Heinzmann, and F. Krausz, Nature (London) 419, 803 (2002).

[4] M. Uiberacker et al., Nature (London) 446, 627 (2007).

[5] E. Goulielmakis et al., Nature (London) 466, 739 (2010).

[6] M. Schultze et al., Science 328, 1658 (2010).

[7] G. Sansone et al., Nature (London) 465, 763 (2010).

[8] F. Kelkensberg et al., Phys. Rev. Lett. 107, 043002 (2011).

[9] A. L. Cavalieri et al., Nature (London) 449, 1029 (2007).

[10] J. Itatani, F. Quéré, G. Yudin, M. Ivanov, F. Krausz, and P. Corkum, Phys. Rev. Lett. 88, 173903 (2002).

[11] R. Kienberger et al., Science 297, 1144 (2002).

[12] P. M. Paul, E. S. Toma, P. Breger, G. Mullot, F. Augé, Ph. Balcou, H. G. Muller, and P. Agostini, Science 292, 1689 (2001).

[13] W. Siu et al., Phys. Rev. A 84, 063412 (2011).

[14] F. Remacle, M. Nest, and R. D. Levine, Phys. Rev. Lett. 99, 183902 (2007).

[15] B. Mignolet, A. Gijsbertsen, M. J. J. Vrakking, R. D. Levine, and F. Remacle, Phys. Chem. Chem. Phys. 13, 8331 (2011).

[16] T. Burnus, M. A. L. Marques, and E. K. U. Gross, Phys. Rev. A 71, 010501 (2005).

[17] A. Castro, H. Appel, M. Oliveira, C. A. Rozzi, X. Andrade, F. Lorenzen, M. A. L. Marques, E. K. U. Gross, and A. Rubio, Phys. Status Solidi B 243, 2465 (2006).

[18] F. Calvayrac, P. Reinhard, and E. Suraud, Phys. Rev. B 52, R17056 (1995); F. Calvayrac, P.-G. Reinhard, E. Suraud, and C. A. Ullrich, Phys. Rep. 337, 493 (2000).

[19] See Supplemental Material at http://link.aps.org/ supplemental/10.1103/PhysRevLett.111.033001 for a description of the experimental setup and the theoretical methodology.

[20] V. Despré et al. (work in progress).

[21] W. Kohn and L. J. Sham, Phys. Rev. 140, A1133 (1965).

[22] P. Johnsson, J. Mauritsson, T. Remetter, A. L'Huillier, and K. Schafer, Phys. Rev. Lett. 99, 233001 (2007).

[23] R. Lopez-Martens et al., Phys. Rev. Lett. 94, 033001 (2005).

[24] A. T. J. B. Eppink and D. H. Parker, Rev. Sci. Instrum. 68, 3477 (1997)

[25] O. Ghafur, W. Siu, P. Johnsson, M. F. Kling, M. Drescher, and M. J. J. Vrakking, Rev. Sci. Instrum. 80, 033110 (2009).

[26] G. U. Bublitz and S. G. Boxer, Annu. Rev. Phys. Chem. 48, 213 (1997). 